\title{
Pace of Aging as a Marker of General
} Health

\section{Current Research in Emergency Medicine (CREM)}

ISSN: 2832-5699

Volume 1, Issue 3, 2021

Article Information

Received date : 23 October, 2021

Published date: 13 December, 2021

*Corresponding author

Fedor Galkin, Deep Longevity Limited, Hong Kong

\section{DOI: $10.54026 / C R E M / 1012$}

\section{Key Words}

COVID-19; Chronological Age; Medical History; DNA Methylation; Geroprotective Therapies

Distributed under: Creative Commons CC-BY 4.0

\section{Fedor Galkin ${ }^{\star}$}

Deep Longevity Limited, Hong Kong

\section{Abstract}

Aging clocks are a type of a statistical model able to estimate the intensity of the basic aging processes based on a set of biomarkers, such as data from blood panels, wearable trackers, psychological survey etc. This technology holds great potential in the healthcare industry due to its diagnostic and prognostic properties.

\section{Opinion}

All people age, but at the same time some people seem to age slower than others. Conversely, others seem to age too fast. This kind of intuition is naturally developed in all humans since we interact with both old and young people on a daily basis. While everyone can use surface-level features (wrinkles, skin tone) to estimate a person's age, only trained professionals have the intuition to tell an old liver and a young liver apart. The task estimating the age of an organ or a system is even harder if you cannot examine it directly.

However, this is not a challenging task for an AI program. Throughout the past decade, dozens of "aging clocks" have emerged. The first of them was published by Steve Horvath in 2013 [1]. Horvath's aging clock uses DNA methylation data that can be obtained from blood (or any other tissue) to detect the fingerprints of aging and quantify the pace of aging. This model has been used to demonstrate that people with various conditions such as Alzheimer's disease, obesity, cancer display accelerated aging [2]. Alternatively, aging clocks can also be used to verify the potential Geroprotective therapies that promise to slow down aging [3].

DNA methylation clocks are widely popular among researchers but the complexity of the underlying sample processing protocol makes them unfit for the clinical settings that require haste. To amend this drawback of the classical molecular aging clocks, our team has developed an aging clock that can process standard blood tests [4]. Accelerated pace of aging it detects has been shown to be a significant risk factor in all-cause mortality [5]. We have also demonstrated that this aging clocks can be used to differentiate smokers and non-smokers [6]. But most importantly, we have shown how this aging clock can benefit the public in the times of the COVID-19 pandemic [7]. The COVID patients that show signs of accelerated aging during admission, according to their blood tests, tend to have lower expected survival time. Note, that the effect of the accelerated aging remains significant in models adjusted for chronological age, symptoms, and medical history.

Blood aging clocks allow for more accurate survival analysis and may be used to efficiently allocate limited resources during a crisis such as the ongoing pandemic.

Conflicts of Interest: None

Financial Support: None

References

1. Horvath S (2013) DNA methylation age of human tissues and cell types. Genome Biol 14(10): R115.

2. Horvath S, Raj K (2018) DNA methylation-based biomarkers and the epigenetic clock theory of ageing. Nat Rev Genet 19(6): 371-384

3. Fitzgerald NK, Hodges R, Hanes D, Stack E, Cheishvili D et al. (2021) Potential reversal of epigenetic age using a diet and lifestyle intervention: a pilot randomized clinical trial. Randomized Controlled Trial 13(7): 9419-9432.

4. Putin E, Mamoshina P, Aliper A, Korzinkin M, Moskalev A, et al. (2016) Deep biomarkers of human aging: Application of deep neural networks to biomarker development. Aging (Albany NY) 8(5): 1021-1033.

5. Mamoshina P, Volosnikova M, Ozerov IV, Putin E, Skibina E et al. (2018) Machine Learning on Human Muscle Transcriptomic Data for Biomarker Discovery and Tissue-Specific Drug Target Identification. Front Genet 9: 242.

6. Mamoshina P, Kochetov K, Cortese F, Kovalchuk A, Aliper A et al. (2019) Blood Biochemistry Analysis to Detect Smoking Status and Quantify Accelerated Aging in Smokers. Sci Rep 9(1): 142.

7. Galkin F, Mamoshina P, Kochetov K, Sidorenko D, Zhavoronkov A (2021) DeepMAge: A Methylation Aging Clock Developed with Deep Learning. Aging Dis 12(5): 1252-1262. 